\title{
Rural-urban differences in dental service use among children enrolled in a private dental insurance plan in Wisconsin: analysis of administrative data
}

\author{
Pradeep Bhagavatula ${ }^{*}$, Qun Xiang ${ }^{2}$, Aniko Szabo², Fredrick Eichmiller ${ }^{3}$, Raymond A Kuthy ${ }^{4}$ and \\ Christopher E Okunseri ${ }^{1}$
}

\begin{abstract}
Background: Studies on rural-urban differences in dental care have primarily focused on differences in utilization rates and preventive dental services. Little is known about rural-urban differences in the use of wider range of dental procedures. This study examined patterns of preventive, restorative, endodontic, and extraction procedures provided to children enrolled in Delta Dental of Wisconsin (DDWI).

Methods: We analyzed DDWI enrollment and claims data for children aged 0-18 years from 2002 to 2008 . We modified and used a rural and urban classification based on ZIP codes developed by the Wisconsin Area Health Education Center (AHEC). We categorized the ZIP codes into 6 AHEC categories (3 rural and 3 urban). Descriptive and multivariable analysis using generalized linear mixed models (GLMM) were used to examine the patterns of dental procedures provided to children. Tukey-Kramer adjustment was used to control for multiple comparisons.

Results: Approximately, 50\%, 67\% and $68 \%$ of enrollees in inner-city Milwaukee, Rural 1 (less than 2500 people), and suburban-Milwaukee had at least one annual dental visit, respectively. Children in inner city-Milwaukee had the lowest utilization rates for all procedures examined, except for endodontic procedures. Compared to children from inner-city Milwaukee, children in other locations had significantly more preventive procedures. Children in Rural 1 -ZIP codes had more restorative, endodontic and extraction procedures, compared to children from all other regions.
\end{abstract}

Conclusions: We found significant geographic variation in dental procedures received by children enrolled in DDWI.

Keywords: Oral health, Urban, Rural, Dental care for children, Dental Insurance, Health services accessibility

\section{Background}

People living in rural and inner-city regions often face significant barriers (such as lack of dental insurance, transportation and shortage of providers) in accessing dental care [1-5], have greater unmet dental need and consequently report poorer oral health status [5-7]. Studies on differences in dental service use in rural and urban areas have primarily focused on enrollees of

\footnotetext{
* Correspondence: pradeep.bhagavatula@mu.edu

${ }^{1}$ Department of Clinical Services, Marquette University School of Dentistry, P.O. Box 1881, Milwaukee, WI 53201-1881, USA

Full list of author information is available at the end of the article
}

public insurance programs and on differences in utilization rates, usually defined as the percentage of population having an annual dental visit [3-5,8]. These studies have reported profound disparities in utilization rates with residents of rural areas having significantly fewer dental visits [3-5,8].

Differences in provision of dental procedures can be used as measures of disparities in dental care. However, very few studies have examined rural vs. urban differences in provision of dental procedures [9-11]. Brennan et al., found that among Australian adults served by public dental programs, those in non-urban areas were 
less likely to have preventive services and more likely to receive restorative, oral surgical and prosthodontic services $[9,10]$. In addition, studies on variation in receipt of dental procedures among children have examined racial differences [12,13], and information on geographic variation in the use of a wider range of dental procedures is nonexistent, especially among the privately insured. This information is important for program planning and policy development aimed at reducing or eliminating differences in dental care if they exist.

Enrollees of private dental insurance plans have higher utilization rates than the uninsured or publicly insured populations [14-16], indicating better access to dental care. Private insurance enrollees have equal access to dental care; however, minorities and people living in low income areas tend to have fewer dental visits $[12,13]$. The association of risk factors in the receipt of dental procedures among children enrolled in private insurance and living in small rural areas and inner-city neighborhoods has not been explored. Identifying these factors is an important step in improving oral health for all enrollees, given that 50 percent of children in the United States are enrolled in private dental insurance plans [14].

In this study we examined the impact of some of the Predisposing, Enabling and Need factors (PEN factors), as described by Andersen et al. [17], on patterns of different dental procedures provided to children living in areas with different levels of urbanization in Wisconsin after adjusting for available covariates such as age and area poverty levels.

\section{Methods}

\section{Data source}

The subjects were enrollees of Delta Dental of Wiscon$\sin$ (DDWI), the largest private insurance dental benefits plan with more than 1.25 million enrollees $(\sim 21 \%$ of people in Wisconsin) [18]. About 90 percent of Wisconsin's dentists are registered providers of DDWI's network. DDWI provides large and small group plans through employers, as well as individual dental plans. It uses network discounted fee schedules for reimbursement of dentists [18].

\section{Measures}

We analyzed enrollment and claims for 0-18 year old children enrolled in DDWI from 2002 to 2008. The enrollment files had information on the number of children insured for each year by ZIP code, gender, and age. The claims dataset had information on age, ZIP code of residence of the child, date of treatment delivery, and procedure code for the treatment provided. We categorized the children into one of five age groups; 0-3 years, 4-6 years, $7-9$ years, $10-14$ years, and $15-18$ years to have a balance in the number of groups as well as the number of enrollees per group, across the various geographic regions. Child age was defined based on the child's age at the last dental visit during the year.

We modified and used a Rural-urban Classification developed by Wisconsin Area Health Education Center (AHEC) to categorize children into six groups (AHEC categories) based on their ZIP code of residence (Table 1) [19], Personal communication from Ms. Nancy Sugden, Wisconsin AHEC]. ZIP codes within rural areas with no population center greater than 2,500 were designated as Rural 1 regions. The ZIP codes within population clusters of 2500-9999 and 10,000-49,999 people were designated as Rural 2 and Rural 3 regions, respectively. Any ZIP codes in areas with population nucleus of 50,000 up to 1 million were designated as 'Urban'. Finally, we modified the classification by adding an additional category to the classification. We categorized ZIP codes in Greater Milwaukee Area (population over 1 million), into inner-city (ICM) and suburban-Milwaukee (SM) groups.

Dental treatment procedures were identified based on Current Dental Terminology (CDT) codes and grouped into six categories. All the diagnostic CDT codes (D0100 to D0999) were categorized as such. CDT codes for oral prophylaxis (D1120), fluoride varnish (D1206), fluoride gel applications (D1203), and dental sealant placement (D1351) were categorized as preventive procedures. Restorative procedures were divided into two categories: simple (D2000 to D2430) and complex restorative procedures (D2510 to D2999). Endodontic procedures included D3000-D3999 and extraction/surgical procedure included codes for extraction of deciduous teeth (D7111), extraction of erupted teeth (D7140) and extraction of erupted teeth requiring elevation of mucoperiosteal flap (D7210).

\section{Conceptual model}

The conceptual framework for this study is closely related to the health behavioral model proposed by Andersen et al, which describes societal and individual determinants of medical care utilization [17]. Under the individual determinants, this model proposes three sets of factors that determine the utilization of healthcare services by individuals. a) predisposing factors which are those that exist prior to disease, and can be either mutable or immutable (b) enabling factors include resources that affect one's ability to access the health care system, and (c) need factors that reflect an illness that requires the use of services. Some of the study variables we used in this study such as; age, rurality or urbanicity place of residence and neighborhood poverty levels are examples of predisposing factors, having private dental insurance, which can also be a proxy for family socioeconomic status, is an enabling factor and the type of treatment 
Table 1 Classification of ZIP codes based on the number of people living in a population cluster+

\begin{tabular}{|c|c|}
\hline AHEC Category & Description \\
\hline Rural 1 & ZIP codes with population of less than $\mathbf{2 5 0 0}$ living in population clusters \\
\hline Rural 2 & ZIP codes with population of $\mathbf{2 , 5 0 0 - 9 , 9 9 9}$ living in population clusters \\
\hline Rural 3 & ZIP codes with population of $\mathbf{1 0 , 0 0 0}-\mathbf{4 9 , 9 9 9}$ living in population clusters \\
\hline Urban & ZIP codes with population of 50,000 up to 1 million living inside urbanized areas outside the Milwaukee metropolitan area \\
\hline Suburban Milwaukee & Milwaukee Metropolitan area; excluding inner-city Milwaukee ZIP codes \\
\hline Urban Milwaukee & Milwaukee county- Inner-city Milwaukee ZIP codes \\
\hline
\end{tabular}

+ Personal Communication from Ms. Nancy Sugden Director, Wisconsin Area Health Education Center.

procedures (e.g., a sealant as opposed to an endodontic procedure) received can be a proxy measure for need.

\section{Statistical analysis}

Descriptive statistics were performed to provide estimates for the number of procedures of each type provided to children across various AHEC categories, and preventive procedures for various age groups. The claims data was aggregated to obtain the number of procedures of each type performed during a calendar year for each enrollee to obtain the averages per year per enrollee. We also calculated the average number of procedures per child per year within each ZIP code based on log transformed ZIP code poverty level to explore the relationship between area poverty and dental procedures. The information on ZIP code poverty levels was obtained from the 2000 US Census information [20]. The overall utilization rates were calculated based on the proportion of enrollees who had at least one dental visit in a given year. Utilization of a specific dental procedure was defined as the presence of a claim for such procedure and utilization rates for treatment procedures were calculated based on the number of enrollees who had at least one procedure in a year.

Multivariable analysis based on Poisson regression with random ZIP code effect was used to test for differences in the number of dental procedures of each type provided to children across the AHEC categories. The covariates in the analysis were age at time of treatment, year of treatment and log transformed poverty levels in the ZIP code. Enrollees from inner-city Milwaukee were used as the reference population for calculating the Rate ratios for provision of dental procedures. The reference group was selected based on initial analysis which showed that they were the group with lowest utilization rates. We also performed pairwise comparisons of average number of procedures between each of the AHEC categories. Tukey-Kramer adjustment was used to control for multiple comparisons. All analyses were performed using SAS version 9.2 (SAS Institute Inc Cary, NC), with PROC GLIMMIX used for the main analysis. A statistical significance level (alpha) of 0.05 was used throughout. This study was approved by the Marquette University's Institutional Review Board.

\section{Results}

The total enrollment during the study period was $1,876,314$ person-years. Table 2 displays the age and gender distribution of enrollees in DDWI during the seven years $(2002$ - 2008). The proportions for the different age groups and gender in all the AHEC categories included in the study were almost equal. The Urban group had the highest and inner-city Milwaukee group had the lowest number of enrollees. The average number of preventive procedures provided per 1000 enrollees is illustrated in Figure 1. The children living in inner-city Milwaukee received the fewest, and those from suburban Milwaukee received the highest number of preventive procedures for most age groups, respectively. Children in $0-3$ and 15-18 age categories from all the AHEC categories received considerably fewer preventive procedures than children in other age groups.

Table 3 reports the utilization rates, and the average number of procedures of each type provided per 1000 enrollees per year for each of the AHEC categories during the study period. Children living in inner-city Milwaukee had fewer dental procedures for most treatment categories included in the analysis except complex restorations and endodontic procedures. Children living in Rural 1 areas received the highest number of corrective procedures (combined restorative, endodontic and extraction), and children from suburban Milwaukee received higher number of preventive procedures.

The proportion of children receiving any preventive procedure increased with the size of the population cluster except for inner-city Milwaukee. Children living in inner-city Milwaukee (35\%), followed by children living in Rural 1 areas (47\%), had the lowest utilization rates for preventive dental procedures. Children living in suburban Milwaukee had the highest utilization rates for preventive services (51\%). A similar pattern was identified for diagnostic procedures where children from inner-city Milwaukee (48\%), followed by those living in Rural 1 areas had lower rates (64\%) and children living in suburban Milwaukee (67\%) had higher rates. Children 
Table 2 Characteristics of study population (0-18 year old children enrolled in DDWI from 2002-2008)

\begin{tabular}{|c|c|c|c|c|c|c|}
\hline & Rural 1 & Rural 2 & Rural 3 & Urban & Suburban Milwaukee & Inner city Milwaukee \\
\hline \multicolumn{7}{|l|}{ Gender } \\
\hline \multirow[t]{2}{*}{ Female } & 162,429 & 129,006 & 87,138 & 336,610 & 137,706 & 48,891 \\
\hline & $(48.37 \%)$ & $(48.16 \%)$ & $(48.60 \%)$ & $(47.50 \%)$ & $(48.62 \%)$ & $(48.14 \%)$ \\
\hline \multirow[t]{2}{*}{ Male } & 169,246 & 136,030 & 90,490 & 356,497 & 141,696 & 51,452 \\
\hline & $(50.40 \%)$ & $(50.78 \%)$ & $(50.47 \%)$ & $(50.31 \%)$ & $(50.03 \%)$ & $(50.67 \%)$ \\
\hline \multirow[t]{2}{*}{ Gender Unknown } & 4,099 & 2,830 & 1,677 & 15,492 & 3,817 & 1,208 \\
\hline & $(1.22 \%)$ & $(1.06 \%)$ & $(0.94 \%)$ & $(2.19 \%)$ & $(1.35 \%)$ & $(1.19 \% \%)$ \\
\hline \multicolumn{7}{|l|}{ Age } \\
\hline \multirow[t]{2}{*}{$0-3$} & 58,138 & 46,602 & 31,523 & 127,904 & 51,226 & 17,540 \\
\hline & $(17.31 \%)$ & $(17.40 \%)$ & $(17.58 \%)$ & $(18.05 \%)$ & $(18.09 \% \%)$ & $(17.27 \%)$ \\
\hline \multirow[t]{2}{*}{$4-6$} & 45,836 & 36,759 & 24,725 & 99,211 & 41,896 & 14,371 \\
\hline & $(13.65 \%)$ & $(13.72 \%)$ & $(13.79 \%)$ & $(14.00 \%)$ & $(14.79 \%)$ & $(14.15 \%)$ \\
\hline \multirow[t]{2}{*}{$7-9$} & 50,522 & 41,406 & 27,142 & 108,701 & 45,355 & 16,005 \\
\hline & $(15.05 \%)$ & $(15.46 \% \%)$ & (15.14\%) & (15.34\%) & (16.01\%) & $(15.76 \% \%)$ \\
\hline \multirow[t]{2}{*}{$10-14$} & 98,850 & 79,119 & 52,561 & 206,399 & 81,356 & 30,031 \\
\hline & (29.44\%\%) & (29.54\%) & (29.31\%) & (29.13\%) & (28.73\%) & $(29.57 \%)$ \\
\hline \multirow[t]{2}{*}{$15-18$} & 82,428 & 63,980 & 43,354 & 166,384 & 63,386 & 23,604 \\
\hline & (24.55\%) & (23.89\%) & $(24.18 \%)$ & $(23.48 \% \%)$ & $(22.38 \%)$ & (23.24\%) \\
\hline Total & 335,774 & 267,866 & 179,305 & 708,599 & 283,219 & 101,551 \\
\hline
\end{tabular}

Numbers in parenthesis represent percentage of the total population.

living in Rural 1 areas had the highest utilization rates for restorative and endodontic procedures of all the groups. Table 3 also shows overall dental utilization rates for enrollees from each of the ZIP code groups. Fifty percent of inner-city Milwaukee enrollees had at least one dental visit. The remaining groups had similar utilization rates $(67-69 \%)$.

Rate ratios from multivariable analysis for comparing dental procedures provided to children across the AHEC categories are summarized in Table 4. Children living in Inner-city Milwaukee were used as the reference group. Compared to children in the reference group, children living in suburban Milwaukee had the highest number of preventive or diagnostic procedure and the fewest number of corrective procedures. Children from Rural 1 areas had the highest number of corrective procedures, with rate ratios of 1.71 and 2.13 for simple and complex restorations and 1.90 and 1.75 for endodontic and extraction procedures, compared to children from innercity Milwaukee. The rates ratios for complex restorative and endodontic procedures for other AHEC groups were not significantly different when compared to the reference population.

Table 4 also reports the rate ratios for procedures with an increase in ZIP code poverty level, and results from pairwise comparisons between the AHEC categories. We

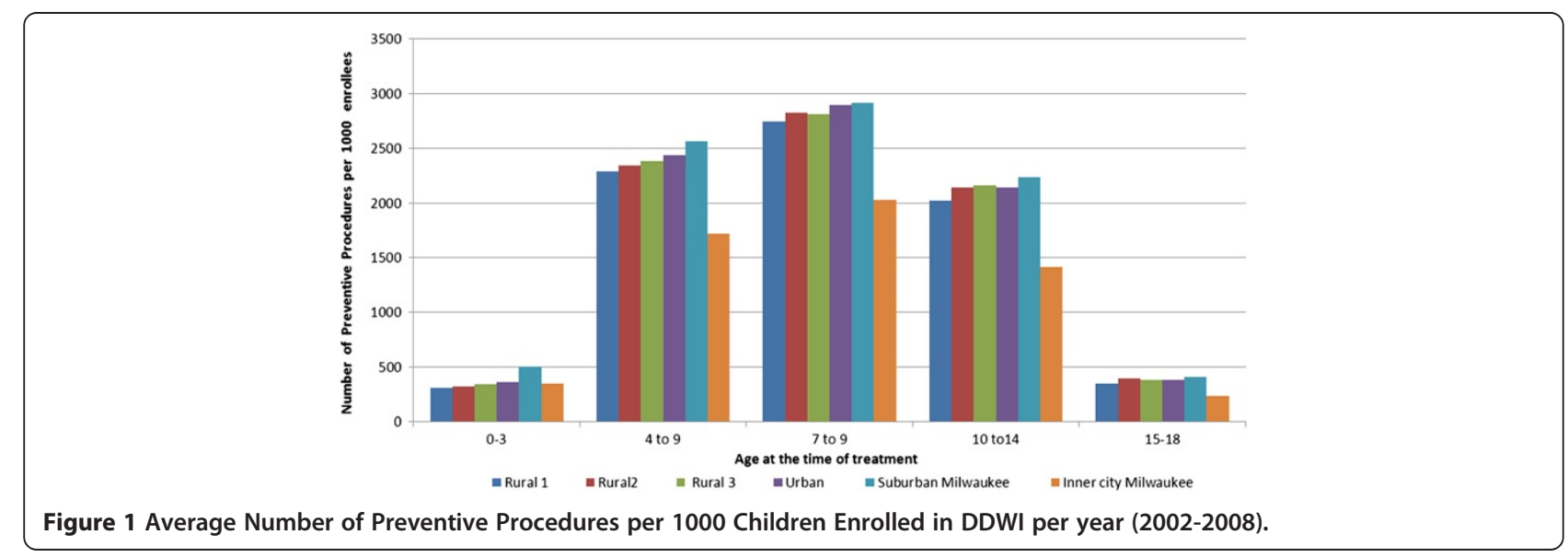


Table 3 Number of dental procedures per 1000 enrollees in a year and utilization rates for dental procedures among children enrolled in DDWI

\begin{tabular}{|c|c|c|c|c|c|c|c|c|c|c|c|c|}
\hline & \multicolumn{2}{|l|}{ Rural 1} & \multicolumn{2}{|l|}{ Rural 2} & \multicolumn{2}{|l|}{ Rural 3} & \multicolumn{2}{|l|}{ Urban } & \multicolumn{2}{|c|}{$\begin{array}{l}\text { Suburban } \\
\text { Milwaukee }\end{array}$} & \multicolumn{2}{|c|}{$\begin{array}{l}\text { Inner city } \\
\text { Milwaukee }\end{array}$} \\
\hline & $\begin{array}{l}\text { No. of } \\
\text { Proc. }\end{array}$ & $\begin{array}{l}\text { Util. } \\
\text { Rate }\end{array}$ & $\begin{array}{l}\text { No. of } \\
\text { Proc. }\end{array}$ & $\begin{array}{l}\text { Util. } \\
\text { Rate }\end{array}$ & $\begin{array}{l}\text { No. of } \\
\text { Proc. }\end{array}$ & $\begin{array}{l}\text { Util. } \\
\text { Rate }\end{array}$ & $\begin{array}{l}\text { No. of } \\
\text { Proc. }\end{array}$ & $\begin{array}{l}\text { Util. } \\
\text { Rate }\end{array}$ & $\begin{array}{l}\text { No. of } \\
\text { Proc. }\end{array}$ & $\begin{array}{l}\text { Util. } \\
\text { Rate }\end{array}$ & $\begin{array}{l}\text { No. of } \\
\text { Proc. }\end{array}$ & $\begin{array}{l}\text { Util. } \\
\text { Rate }\end{array}$ \\
\hline Diagnostic & 1653 & $64.0 \%$ & 1703 & $66.0 \%$ & 1666 & $66.0 \%$ & 1750 & $67.0 \%$ & 1749 & $67.0 \% \%$ & 1314 & $48.0 \%$ \\
\hline Preventive & 1477 & $47.0 \%$ & 1556 & $49.0 \%$ & 1554 & $48.0 \%$ & 1599 & $50.0 \%$ & 1694 & $51.0 \%$ & 1109 & $35.0 \%$ \\
\hline Simple Restorative & 558 & $21.0 \%$ & 511 & $20.0 \% \%$ & 503 & $20.0 \%$ & 442 & $18.0 \%$ & 421 & $17.0 \%$ & 362 & $14.0 \%$ \\
\hline Complex Restorative & 35 & $2.0 \%$ & 31 & $2.0 \% \%$ & 28 & $2.0 \%$ & 30 & $2.0 \%$ & 26 & $1.0 \%$ & 29 & $2.0 \%$ \\
\hline Endodontic & 31 & $2.0 \%$ & 28 & $1.0 \%$ & 28 & $1.0 \%$ & 28 & $1.0 \%$ & 23 & $1.0 \%$ & 29 & $2.0 \%$ \\
\hline Extractions & 82 & $4.0 \%$ & 78 & $4.0 \%$ & 75 & $3.0 \%$ & 84 & $4.0 \%$ & 73 & $3.0 \%$ & 61 & $3.0 \%$ \\
\hline $\begin{array}{l}\text { Corrective (Restorative + } \\
\text { Endodontic + Extractions) }\end{array}$ & 706 & - & 648 & - & 634 & - & 584 & - & 543 & - & 481 & - \\
\hline Total/Overall Utilization & 3866 & $67.0 \%$ & 3907 & $68.0 \% \%$ & 3854 & $68.0 \%$ & 3933 & $69.0 \%$ & 3986 & $68.0 \%$ & 2904 & $50.0 \%$ \\
\hline
\end{tabular}

No. of Proc- Number of procedures per 1000 years of enrollment; Util. Rate- Utilization rate for a given procedure i.e. proportion of children receiving at least one procedure in an year; Total/Overall Utilization: proportion of children with at least one annual dental visit.

found that as the ZIP code poverty levels increase, there was a decrease in the number of diagnostic and preventive procedures and an increase in corrective and extraction procedures. In the pairwise comparisons analysis we found that children from Rural 1 and inner-city Milwaukee groups were significantly different from the other AHEC groups for most treatment procedures. For the remaining AHEC categories we found that Rural 2 and Rural 3 were similar to each other, and Urban and suburban Milwaukee groups were similar to each other for most procedures examined.

\section{Discussion}

In this study we examined the patterns of utilization of dental services and procedures among 0-18 year old enrollees in DDWI from 2002 to 2008. The subjects are residents of areas with different levels of urbanization in the state of Wisconsin. To our knowledge this is the first study to compare dental procedures provided to privately insured children living in inner-city and rural areas.

We found that the utilization rates for children from all geographic regions were similar except for those from inner-city Milwaukee, who had much lower rates. These findings are different from previous studies which reported significant differences in utilization rates between rural and urban populations $[3,5,8]$. In this study, approximately, $67 \%, 68 \%$ and $50 \%$ of children from small rural communities, suburban Milwaukee and inner city Milwaukee had at least one annual dental visit, respectively. These rates are lower than the rates reported for rural $(69.9 \%)$ and urban (73.6\%) children by Vargas et al., who analyzed the data from National Health Interview Survey (NHIS) and National Health and Nutrition Examination Survey (NHANES) [5] which are based on representative samples of the United States and include

Table 4 Results from multivariable analysis examining geographic variation in dental procedures among 0-18 year old children enrolled in DDWI from 2002-2008 and the impact of ZIP code level poverty

\begin{tabular}{|c|c|c|c|c|c|c|c|}
\hline & Ref. Group $\$$ & $\begin{array}{l}\text { Impact of } \\
\text { Poverty }^{+}\end{array}$ & $\begin{array}{l}\text { Impact of } \\
\text { Poverty }^{+}\end{array}$ & $\begin{array}{l}\text { Impact of } \\
\text { Poverty }^{+}\end{array}$ & $\begin{array}{l}\text { Impact of } \\
\text { Poverty }^{+}\end{array}$ & $\begin{array}{l}\text { Impact of } \\
\text { Poverty }^{+}\end{array}$ & $\begin{array}{l}\text { Impact of } \\
\text { Poverty }^{+}\end{array}$ \\
\hline & $\begin{array}{l}\text { Inner-city } \\
\text { Milwaukee }\end{array}$ & Rural 1 & Rural 2 & Rural 3 & Urban & $\begin{array}{l}\text { Suburban } \\
\text { Milwaukee }\end{array}$ & \\
\hline Diagnostic & $1.00 \mathrm{a}$ & $1.20(1.12-1.29) b$ & $1.26(1.17-1.36) b c$ & $1.27(1.16-1.38) b c$ & $1.26(1.17-1.36) b c$ & $1.25(1.15-1.36) c$ & $0.95(0.94-0.96)$ \\
\hline Preventive & $1.00 \mathrm{a}$ & $1.29(1.17-1.42) b$ & $1.37(1.24-1.52) b c$ & $1.45(1.28-1.63) c$ & $1.38(1.25-1.52) c$ & $1.40(1.25-1.57) b c$ & $0.93(0.92-0.94)$ \\
\hline $\begin{array}{l}\text { Simple } \\
\text { Restorative }\end{array}$ & $1.00 \mathrm{a}$ & $1.71(1.53-1.91) b$ & 1.59(1.41-1.79)b & $1.51(1.31-1.73) b c$ & $1.37(1.21-1.54) \mathrm{c}$ & $1.30(1.14-1.48) \mathrm{C}$ & $1.01(1.00-1.03)$ \\
\hline $\begin{array}{l}\text { Complex } \\
\text { Restorative }\end{array}$ & $1.00 \mathrm{a}$ & $2.13(1.68-2.71) b$ & $1.44(1.10-1.88) \mathrm{ac}$ & $1.29(0.95-1.75) \mathrm{ac}$ & $1.51(1.17-1.96) c$ & 1.39 (1.04- 1.87) ac & $1.20(1.15-1.25)$ \\
\hline Endodontic & $1.00 \mathrm{a}$ & $1.90(1.53-2.35) b$ & $1.33(1.05-1.68) a$ & $1.26(0.97-1.65) a$ & $1.39(1.11-1.74) a$ & $1.20(0.93-1.55) a$ & $1.14(1.10-1.19)$ \\
\hline Extractions & $1.00 \mathrm{a}$ & $1.75(1.53-2.00) b$ & $1.52(1.31-1.75) \mathrm{C}$ & $1.50(1.27-1.77) \mathrm{C}$ & $1.58(1.37-1.81) c$ & $1.51(1.29-1.77) \mathrm{C}$ & $0.99(0.96-1.03)$ \\
\hline
\end{tabular}


both publicly and privately insured children. They also reported utilization rates of $79.3 \%$ and $80.1 \%$ for rural and urban children from non-poor families (at or over $200 \%$ of the FPL), respectively, [5] which are considerably higher than the rates we found in this privately insured population.

In the analyses comparing receipt of dental procedures, we found that children living in small rural and inner-city areas have fewer preventive and diagnostic procedures. We also found a concomitant increase in use of these procedures and urbanization. Similarly, there was a decrease in the utilization of these procedures with increasing poverty levels in the ZIP codes. The subjects in this study have $100 \%$ coverage for diagnostic and preventive procedures, but we still found significant differences in use of these procedures suggesting that the level of urbanization and area poverty play a role in dental access and utilization even in these insured populations.

Compared to children living in other areas, we found that enrollees from inner-city had the lowest, and those from small rural areas had the highest number of restorative and endodontic procedures, respectively. Previous studies reported that rural residents, racial/ethnic minorities and individuals from low socioeconomic backgrounds are more likely to visit a dentist because of a problem or when in pain, necessitating complex restorations, endodontic procedures and extractions $[5,12,21,22]$. We found that children from Rural 1 regions had fewer visits than children from more urbanized areas, yet they have the highest number of corrective procedures of all groups included in the analysis. Our findings suggest that children in this group may have higher disease levels and/or an episodic pattern of care. On the contrary, children from inner-city region had the fewest procedures of all types when compared to other children. While low overall dental utilization rates for children in this group may explain this finding, it also suggests that they may be facing additional barriers [14] or have higher levels of untreated disease.

Socio-economic status of an individual and his or her place of residence are closely related and have been shown to independently affect the person's health, access to healthcare and health outcomes $[6,23,24]$. People living in areas with higher poverty levels have fewer preventive care visits and procedures compared to those who live in high income areas [25-27]. These disparities have been shown to persist even after adjusting for factors such as insurance status, differences in supply of providers, and having regular source of care [25]. The children in this study are enrolled in a private dental insurance plan and are not random representatives of their ZIP codes in terms of poverty. Nonetheless, we found that within each AHEC category, as ZIP code poverty levels increased, there was a decrease in the number of diagnostic and preventive procedures and an increase in endodontic and surgical procedures (data not show). We found a similar pattern from the multivariable analysis showing that ZIP code poverty is an independent predictor of dental care patterns even in this privately insured population.

The utilization rates for all procedures examined in the analysis were lowest among children from inner-city Milwaukee and the poverty levels were among the highest in these ZIP codes. This combination has led to a situation wherein the rate ratios for the treatment procedures from multivariable analysis remain high as opposed to being closer to null after controlling for poverty. This, we suspect, is because of negative confounding. As we projected the low-usage rate of a high poverty group (inner-city Milwaukee) to higher usage rate of groups with lower poverty, the usage of these procedures is expected to be lower than what is actually observed. In other words, since these areas did not have such high poverty levels, the model would expect their rate of usage of these procedures to be much lower than the actual values. This widens the gap between the groups leading to an increase in the magnitude of the rate ratios for these procedures after adjusting for poverty.

\section{Strengths and limitations}

We determined relative rurality or urbanicity of the location of residence using population size living in an urban cluster. While this approach has its drawbacks, it overcomes the limitations of previous methods used for classification. The Rural Urban Commuting Area (RUCA) system, [28] Urban Influence codes [29] and dichotomization into rural and urban areas are among the more commonly used methods for making this classification. The main limitation of the RUCA system is that it is based on commuting patterns which categorizes many non-suburban metro-adjacent areas in the "metropolitan" category. Urban Influence codes are county level measures based on the largest metropolitan area and may misclassify large number of people who live in rural areas within those counties. Using the UIC method will categorize over $30 \%$ of the rural populations in the state of Wisconsin as "metropolitan" dwelling [Personal communication from Wisconsin AHEC]. The strategy used by us uses census tract data summarized at the level of place to classify cities, villages and towns at different levels as urban or rural. This strategy would also differentiate large metropolitan areas like Milwaukee from other metropolitan areas, as well as inner-city and suburban Milwaukee areas.

There are limitations to our study that should be noted. First, our dataset did not include child-level 
information on dental need but only procedure billing codes. Second, our final statistical models did not include factors that have been shown to be associated with patterns of dental care utilization such as availability of providers, parental education and perceptions of need, and family socioeconomic status, however, we employed proxy measures by disaggregating this information from ZIP codes. Our results do seem to suggest that at least shortage of providers is not a significant barrier to care in this population, as we found identical utilizations rates for all groups and the only group with lower rates (inner-city Milwaukee) is close to areas with dental providers. Third, we were unable to examine whether some of the extractions were due to orthodontic treatment, however, it is unlikely that the high rates of extractions in Rural 1 areas would be explained by orthodontic treatments. Finally, our results have limited generalizability because only data for children enrolled in one private dental insurance carrier was analyzed, albeit the largest in the state.

\section{Conclusions}

We found significant geographic variations in dental procedures received by children enrolled in DDWI. Inner-city Milwaukee children had significantly fewer preventive and diagnostic dental procedures and those from the smallest rural communities had higher rates of all other dental procedures.

\section{Competing interests}

None of the authors have any financial or Non-financial competing interests in the project. FE is employed by Delta Dental of Wisconsin but declares no competing interest.

\section{Authors' contribution}

PB participated in design, acquisition of data, interpretation of the results, and took the lead in writing the manuscript. QX and AS performed the statistical analysis and interpretation of the results. FE helped in acquisition of the data. FE, RK and CO contributed to completion of the manuscript and have critically revised the manuscript. All authors have read and approved the final manuscript will hold themselves jointly and individually responsible for its content.

\section{Acknowledgement}

We wish to thank Ms. Nancy Sugden, Director of Wisconsin Area Health Education Center, for providing us with the Rural- Urban classification of Wisconsin ZIP codes for use in this project.

\section{Funding}

This project was supported, in part, by grant 1UL1RR031973 from the Clinical and Translational Science Award (CTSI) program of the National Center for Research Resources, National Institutes of Health.

\section{Author details}

${ }^{1}$ Department of Clinical Services, Marquette University School of Dentistry, P.O. Box 1881, Milwaukee, WI 53201-1881, USA. ²Division of Biostatistics, Institute for Health and Society, Medical College of Wisconsin, Milwaukee, USA. ${ }^{3}$ Delta Dental of Wisconsin, Milwaukee, USA. ${ }^{4}$ Department of Preventive and Community, Dentistry University of lowa College of Dentistry,

Milwaukee, USA.
Received: 16 March 2012 Accepted: 13 December 2012

Published: 21 December 2012

\section{References}

1. DeNavas-Walt C, Proctor BD, Smith JC, U.S. Census Bureau: Current Population Reports, P60-235, Income, Poverty, and Health Insurance Coverage in the United States. Washington, DC: U.S. Government Printing Office; 2008.

2. Isman R, Isman BE: Oral Health America White Paper: Access to Oral Health Services in the United States 1997 and Beyond. Chicago, III: Oral Health America, America's Fund for Dental Health; 1998.

3. Byck GR, Walton SM, Cooksey JA: Access to dental care services for Medicaid children: Variations by urban/rural categories in Illinois. J Rural Health 2002, 18:512-520.

4. Vargas CM, Dye BA, Hayes K: Oral health care utilization by us rural residents, national health interview survey 1999. J Public Health Dent 2003, 63:150-157.

5. Vargas CM, Ronzio CR, Hayes KL: Oral health status of children and adolescents by rural residence, united states. J Rural Health 2003, 19:260-268.

6. Tellez M, Sohn W, Burt BA, Ismail Al: Assessment of the relationship between neighborhood characteristics and dental caries severity among low-income African-Americans: a multilevel approach. J Public Health Dent 2006, 66(1):30-6.

7. Sohn W, Ismail A, Amaya A, Lepkowski J: Determinants of dental care visits among low-income African-american children. J Am Dent Assoc 2007, 138:309-318. quiz 395-396, 398

8. Allison RA, Manski RJ: The supply of dentists and access to care in rural Kansas. J Rural Health 2007, 23(3):198-206.

9. Brennan DS, Spencer AJ, Slade GD: Provision of public dental services in urban, rural and remote locations. Community Dent Health 1996, 13(3):157-62.

10. Brennan D, Spencer AJ, Szuster F: Rates of dental service provision between capital city and non-capital locations in Australian private general practice. Aust J Rural Health 1998, 6(1):12-7.

11. Okada $L M$, Sparer $G$ : Dental visits by income and race in ten urban and two rural areas. Am J Public Health 1976, 66(9):878-85.

12. Bhagavatula $P Q$, Xiang A, Szabo F, Eichmiller CE: Okunseri Racial Disparities in Provision of Dental Procedures to Children Enrolled in Delta Dental Insurance in Milwaukee, Wisconsin. J Public Health Dent, . in press.

13. Macek MD, Edelstein BL, Manski RJ: An analysis of dental visits in U.S. children, by category of service and sociodemographic factors, 1996. Pediatr Dent 2001, 23(5):383-389.

14. Lewis C, Mouradian W, Slayton R, Williams A: Dental insurance and its impact on preventive dental care visits for U.S. children. J Am Dent Assoc 2007, 138(3):369-80.

15. Grembowski D, Conrad D, Milgrom P: Utilization of dental services in the United States and an insured population. Am J Public Health 1985, 75 (1):87-9.

16. Manski RJ, Edelstein BL, Moeller JF: The impact of insurance coverage on children's dental visits and expenditures, 1996. J Am Dent Assoc 2001, 132(8):1137-45.

17. Andersen R, Newman JF: Societal and individual determinants of medical care utilization in the United States. Milbank Mem Fund Q Health Soc 1973, 51(1):95-124.

18. About Delta Dental of Wisconsin: [http://www.deltadentalwi.com/ aboutdeltawi] [Accessed on September, 14 2012].

19. [http://www.worh.org/WisRuralAreas] [Accessed on September, 14 2012]

20. Zip Codes Percentage of Families Below Poverty Level in Wisconsin. http://zipatlas.com/us/wi/zip-code-comparison/families-below-poverty-level. htm.

21. Vargas CM, Ronzio CR: Relationship between children's dental needs and dental care utilization: United States, 1988-1994. Am J Public Health. 2002, 92(11):1816-21.

22. Riley JL, Gilbert GH, Heft MW: Orofacial pain: Patient satisfaction and delay of urgent care. Public Health Rep. 2005, 120:140-149.

23. General Accounting Office. Oral Health: Factors Contributing to Low Use of Dental Services by Low-Income Populations; U.S. General Accounting Office, Report to Congressional Requesters. HEHS-00-149. 2000. Sep 11.

24. Thomson WM, Mackay TD: Child dental caries patterns described using a combination of area-based and household-based socio-economic status measures. Community Dent Health. 2004, 21(4):285-90. 
25. Kirby JB, Kaneda T: Neighborhood socioeconomic disadvantage and access to health care. J Health Soc Behav. 2005, 46(1):15-31.

26. Larson SL, Correa-de-Araujo R: Preventive health examinations: A comparison along the rural-urban continuum. Womens Health Issues; 2006, 16:80-88.

27. Larson SL, Fleishman JA: Rural-urban differences in usual source of care and ambulatory service use: Analyses of national data using urban influence codes. Med Care 2003, 41:|l|65-III74.

28. Morrill R, Cromartie J, Hart G: Metropolitan, Urban, and Rural Commuting Areas: Toward a Better Depiction of the United States Settlement System. Urban Geography 1999, 20:727-748.

29. Economic Research Service, US Department of Agriculture: Accessed at http://www.ers.usda.gov/briefing/rurality/urbaninf/ on 2011.

doi:10.1186/1472-6831-12-58

Cite this article as: Bhagavatula et al:: Rural-urban differences in dental service use among children enrolled in a private dental insurance plan in Wisconsin: analysis of administrative data. BMC Oral Health 2012 12:58.

\section{Submit your next manuscript to BioMed Central and take full advantage of:}

- Convenient online submission

- Thorough peer review

- No space constraints or color figure charges

- Immediate publication on acceptance

- Inclusion in PubMed, CAS, Scopus and Google Scholar

- Research which is freely available for redistribution 